\title{
Changing trend of antimicrobial resistance among gram-negative bacilli isolated from lower respiratory tract of ICU patients: A 5-year study
}

\author{
Deep Gagneja, Nidhi Goel, Ritu Aggarwal, Uma Chaudhary
}
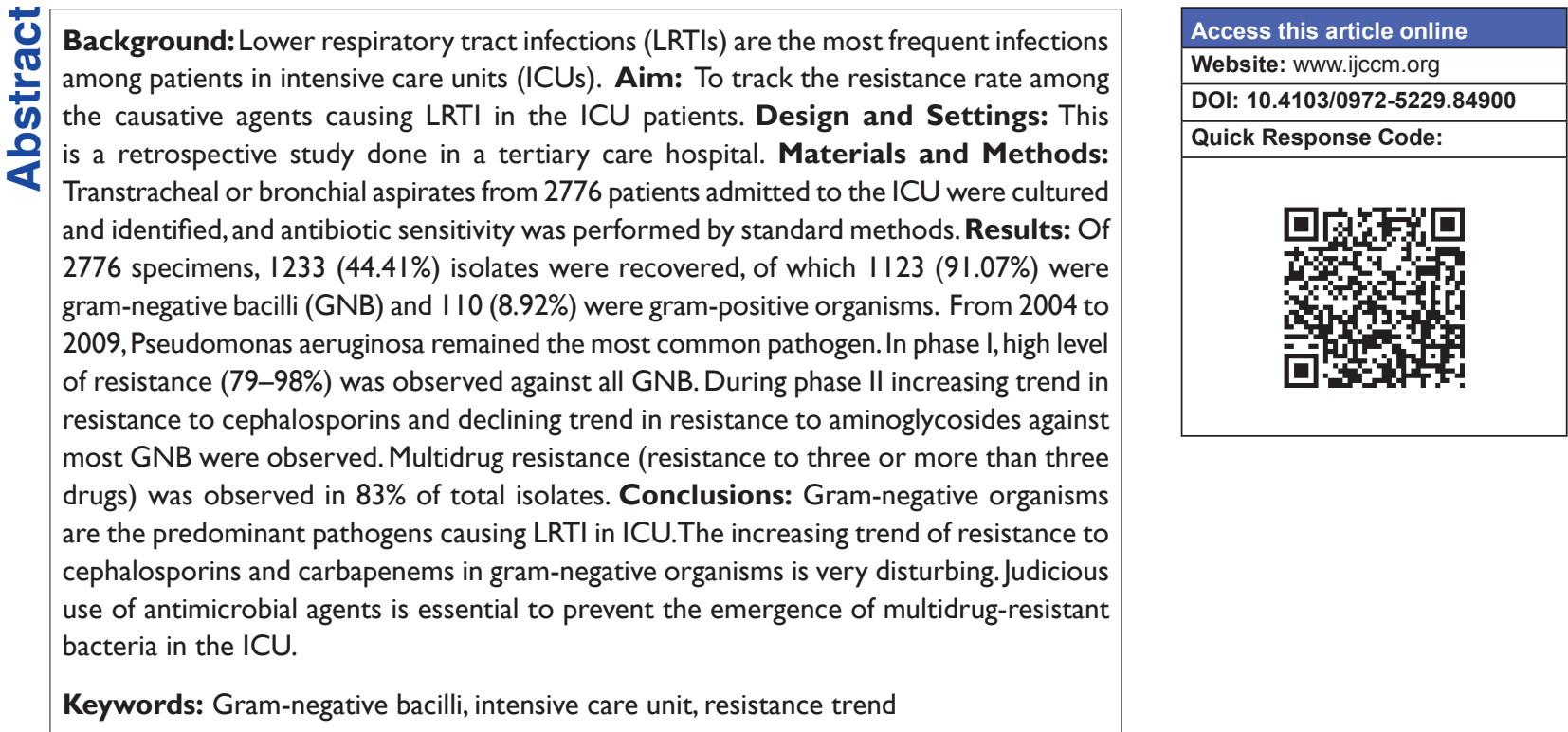

\section{Introduction}

Lower respiratory tract infections (LRTI) are the most common bacterial infections among patients in intensive care units (ICUs), occurring in $10-25 \%$ of all ICU patients and resulting in high overall mortality, which may range from 22 to $71 \% \cdot{ }^{[1,2]}$ A multicenter study conducted in 13 European countries indicated that respiratory tract samples (39\%) were the most frequent source of bacterial isolates followed by blood (21\%) and urinary tract $(17 \%) \cdot{ }^{[3]}$ Most common bacterial agents of

From:

Department of Microbiology, Pandit Bhagwat Dayal Sharma Post Graduate Institute of Medical Sciences, Rohtak, India

Correspondence:

Dr. Nidhi Goel, Department of Microbiology, Pandit Bhagwat Dayal Sharma Post Graduate Institute of Medical Sciences, Rohtak, India.

E-mail: ngoel_2003@yahoo.com
LRTI in the ICU are Pseudomonas, Acinetobacter, Klebsiella, Citrobacter, and Escherichia coli. ${ }^{[4-6]}$ The incidence and associated mortality due to LRTI can be influenced by several factors including characteristics of the population at risk, standard of the healthcare facilities available, immunosuppressive drugs, inappropriate antibiotic therapy, distribution of causative agents, and prevalence of antimicrobial resistance. These factors may vary from one geographical region to the other. In almost all cases, eradication of causative agents requires initiation of antimicrobial therapy before obtaining culture report; however, during the last few years, the increase in antibiotic resistance has compromised the selection of empirical treatment. ${ }^{[7]}$ The knowledge of likely prevalent strains along with their antimicrobial resistance pattern will help in better management of patients and framing the antibiotic policy. This study was conducted with 
the aim of tracking resistance rate among the causative agents of LRTI in ICU patients.

\section{Materials and Methods}

The present retrospective study was conducted at the Microbiology Department of a teaching tertiary care hospital over a period of 5 years (July 2004-July 2009). Transtracheal or bronchoscopic aspirates were collected aseptically from the patients of all age groups and sex admitted to respiratory intensive care unit, who were on mechanical ventilation for at least 3 days. Samples of only new patients who were enrolled for the first time were included in the study. Single or mixed growth from one patient and consecutive samples from the new patients were included in the study. If the repeat sample was received from the same patient who was already enrolled, it was not included in the study. All the specimens received were immediately plated on the blood agar and Mac-Conkey agar by semiquantitative method and incubated aerobically overnight at $37^{\circ} \mathrm{C}$. Organisms were identified as commensal or pathogen as per protocol. Tracheal aspirates showing less than $10^{5} \mathrm{cfu} / \mathrm{ml}$ and bronchial secretions with less than $10^{4}$ $\mathrm{cfu} / \mathrm{ml}$ by semiquantitative culture were regarded as commensal or contaminant.

Single or mixed growth (two or more than two isolates per specimen) isolated from all the eligible consecutive samples were identified by observing the colony characteristic on the blood, Mac-Conkey agar plate, and biochemical reactions using standard microbiological methods. ${ }^{[8]}$ Isolates from repeat culture of previously recruited patients and isolates identified as commensal or contaminants were excluded. The study was conducted in two phases: phase I (2004-6) and phase II (2006-9). During phase I, activity of ampicillin, cefuroxime, gentamicin, ciprofloxacin, cefotaxime, amikacin, and ceftizoxime was tested. During phase II, activity of ceftriaxone, ceftazidime, ceftizoxime, amoxy-clav, gentamicin, amikacin, ciprofloxacin, meropenem, piperacillin, piperacillin tazobactom, aztreonam, netilmycin, and ofloxacin was tested. Susceptibility testing was done by disc diffusion method. ${ }^{[9]}$ The following antibiotics (Hi-Media Disc in $\mu \mathrm{g}$ ) were tested: ampicillin (10), piperacillin (100), ceftazidime (Cz) (30), ceftizoxime (Ck) (30), cefuroxime (30), cefotaxime (30), ceftriaxone (30), amoxycillin/clavulanic acid (Ac) (20/10), piperacillin/ tazobactum (TZP) (100/10), aztreonam (Ao) (30) , meropenem (Mr) (10), gentamicin (10), amikacin (Ak) (30), ciprofloxacin (Cf) (5), ofloxacin (Of) (5), and imipenem(I) (10). Zone diameter was measured and interpreted as per the Clinical and Laboratory Standards Institute guidelines. For quality control of disc diffusion tests, ATCC control strains of E. coli ATCC 25922 and Pseudomonas aeruginosa ATCC 27853 strains were used.

\section{Results}

During the study period, laboratory data of 2776 patients whose LRTI specimens were received in our laboratory were evaluated. Male to female ratio was 1:1. About $21.61 \%$ of patients were in age group of less than 17 years, $42.15 \%$ in $18-64$ years, and $36.38 \%$ were of more than 65 years of age. Out of 2776 specimens, 1125 (40.52\%) were culture positive, whereas 1651 (59.47\%) specimens showed no growth. Out of 2776 specimens, 1233 (44.41\%) isolates were recovered. A total of 1017 samples showed single isolation, whereas from 108 samples, 2 isolates per specimen were recovered. Out of the total 1233 isolates identified, 1123 (91.07\%) were gram-negative bacilli (GNB) and $110(8.92 \%)$ were gram-positive organisms. Aerobic GNB remained the predominant cause of LRTI in ICU patients throughout the study period.

Table 1 shows year-wise distribution of GNB isolated from the lower respiratory tract. During the 5-year span, $P$. aeruginosa remained the most common pathogen. Trends of antimicrobial resistance among the leading pathogens causing LRTI were studied in two phases, i.e., from 2004-2006 (phase I) and 2006-2009 (phase II). Pattern of antimicrobial resistance in phase I is shown in Table 2. During the second phase, few new antimicrobials were added in the panel. Phase II showed a increasing trend in resistance to cephalosporins and declining trend in resistance to aminoglycosides against most GNB.

\begin{tabular}{|c|c|c|c|c|c|}
\hline Organisms & $\begin{array}{c}2004-2005 \\
\text { n (246) }\end{array}$ & $\begin{array}{c}2005-2006 \\
(2 \mid 4)\end{array}$ & $\begin{array}{c}2006-2007 \\
(I 85)\end{array}$ & $\begin{array}{c}2007-2008 \\
(154)\end{array}$ & $\begin{array}{c}2008-2009 \\
(324)\end{array}$ \\
\hline P. aeruginosa & $96(39.02)$ & $107(50)$ & $68(36.75)$ & $57(39.58)$ & $96(29.62)$ \\
\hline Klebsiella spp. & $66(26.82)$ & $18(8.41)$ & $29(15.67)$ & $22(14.28)$ & $53(16.35)$ \\
\hline Acinetobacter spp. & $29(11.98)$ & 38 (I7.95) & $44(23.78)$ & $38(26.37)$ & $81(25)$ \\
\hline E. coli & $7(2.84)$ & $16(7.47)$ & $14(7.56)$ & $12(7.79)$ & $30(9.25)$ \\
\hline Enterobacter spp. & $26(10.56)$ & 27 (I2.6I) & $23(12.43)$ & I7 (I I.03) & $35(10.80)$ \\
\hline Citrobacter spp. & $21(8.53)$ & 7 (3.27) & $6(3.24)$ & $8(5.19)$ & $29(8.95)$ \\
\hline Proteus spp. & I $(0.40)$ & I $(0.46)$ & I $(0.54)$ & 0 & 0 \\
\hline
\end{tabular}

Figures in parentheses indicate percentage, GNB = Gram-negative bacilli, ICU = Intensive care units. 
Sensitivity to quinolones was relatively stable among GNB [Table 3]. In 2006-2007, resistance to ceftriaxone and ceftazidime ranged from 66 to $97 \%$ and it increased to 80 to $97 \%$ in 2008-2009. But in contrast to other gramnegative isolates, Acinetobacter isolates were more resistant to ceftriaxone, ceftizoxime, and ceftazidime (85-97\% in 2006-207), but it also increased to 95-97\% in 2008-2009. As far as resistance to aminoglycosides is concerned, resistance to gentamicin was relatively high when compared with amikacin in all the 3 years of phase II. But resistance to both these drugs decreased continuously during phase II [G, 66-79\% (2006-2007) to 51-57\% (2008-2009)], [AK, 50-65\% (2006-2007) to 48-53\% (2008-2009)]. About 93\% of Acinetobacter isolates showed resistance to gentamicin in 2006-2007 which reduced to $72.83 \%$ in 2008-2009. In contrast, Acinetobacter isolates showed increasing trend of resistance to amikacin (65.9-70.37\%). Multidrug resistance (resistance to three or more than three drugs) was observed in $83 \%$ of total isolates during study period.

\section{Discussion}

In this study, GNB were the predominant organisms causing LRTI. During the study period of the 5 years, $P$. aeruginosa remained the most common organism (30-50\%) followed by Klebsiella spp., but from 2005 to 2006 onward, the rate of isolation of Acinetobacter spp. increased from $11.78 \%$ (2004-2005) to $25 \%$ (2008-2009) becoming the second most common isolate. Lokhart et al[10] reported $P$. aeruginosa as the most common isolate from lower respiratory tract specimens in ICU, but Kumari et al ${ }^{[11]}$ reported non-fermenting GNB other than $P$. aeruginosa as the most common pathogens. During phase I, high level of resistance was observed to ampicillin (97-98\%), cefuroxime (90-93\%), gentamicin $(79-80 \%)$, and amikacin (70-71\%) against all the GNB, so during phase II some new drugs such as ceftriaxone, ceftazidime, and amoxicillin-clavulanic acid were added to the panel for GNB other than P. aeruginosa. For $P$. aeruginosa, other additional drugs tested were ofloxacin, piperacillin, piperacillin/tazobactam, netilmycin, and aztreonam. We observed an alarming increasing trend

\begin{tabular}{lccccccc}
\hline \multicolumn{7}{l}{ Table 2: Trends in antimicrobial susceptibility pattern of GNB during 2004-2006 } & \\
\hline & Ampicillin & Cefuroxime & Gentamicin & Ciprofloxacin & Cefotaxime & Amikacin & Ceftizoxime \\
\hline $2004-2005(n=246)$ & $240(97.56)$ & $216(90)$ & $197(80.08)$ & $188(76.42)$ & $177(71.95)$ & $175(71.11)$ & $157(63.82)$ \\
$2005-2006(n=214)$ & $208(97.19)$ & $193(92.78)$ & $170(79.43)$ & $151(72.59)$ & $150(70.09)$ & $145(69.71)$ & $135(64.9)$ \\
\hline
\end{tabular}

GNB = Gram-negative bacilli.

\begin{tabular}{|c|c|c|c|c|c|c|c|c|c|c|c|c|c|}
\hline & CTX & CTa & CTi & AC & G & AK & CF & Mr & PC & PT & $\mathbf{A z}$ & NT & Of \\
\hline \multicolumn{14}{|l|}{ E. coli } \\
\hline 2006-2007 (I4) & 78.51 & 85.7 & 50 & 71.42 & 78.57 & 57.14 & 71.42 & 21.4 & & & & & \\
\hline $2007-2008$ (I 2$)$ & 75 & 83.33 & 83.33 & 66.66 & 75 & 58.33 & 66.66 & 58.33 & & & & & \\
\hline 2008-2009 (30) & 93.33 & 90 & 90 & 90 & 53.33 & 53.33 & 73.33 & 56.66 & & & & & \\
\hline \multicolumn{14}{|l|}{ Klebsiella spp. } \\
\hline 2006-2007 (29) & 72.41 & 82.79 & 75.86 & 62.05 & 75.86 & 58.62 & 82.75 & 13.7 & & & & & \\
\hline 2007-2008 (22) & 81.81 & 90.90 & 90.90 & 72.72 & 72.72 & 63.63 & 81.81 & 50 & & & & & \\
\hline $2008-2009(53)$ & 94.33 & 96.22 & 94.33 & 88.67 & 56.6 & 58.49 & 79.24 & 56.6 & & & & & \\
\hline \multicolumn{14}{|l|}{ Enterobacter spp. } \\
\hline 2006-2007 (23) & 73.91 & 82.60 & 65.21 & 65.21 & 86.95 & 65.21 & 78.26 & 8.69 & & & & & \\
\hline $2007-2008$ (I7) & 88.23 & 88.23 & 88.25 & 76.47 & 64.7 & 58.82 & 76.47 & 47.05 & & & & & \\
\hline 2008-2009 (35) & 94.28 & 94.28 & 91.42 & 85.71 & 54.28 & 51.42 & 85.7I & 45.7I & & & & & \\
\hline \multicolumn{14}{|l|}{ Citrobacter spp. } \\
\hline 2006-2007 (6) & 66.66 & 83.33 & 50 & 50 & 66.66 & 50 & 66.66 & 16.6 & & & & & \\
\hline $2007-2008$ (8) & 8.75 & 75 & 75 & 62.5 & 62.5 & 50 & 75 & 37.5 & & & & & \\
\hline 2008-2009 (29) & 82.75 & 79.31 & 82.75 & 82.75 & 51.72 & 48.27 & 75.86 & 65.51 & & & & & \\
\hline \multicolumn{14}{|l|}{ Proteus spp. } \\
\hline 2006-2007 (I) & - & - & 100 & - & - & - & 100 & - & & & & & \\
\hline $2007-2008(0)$ & - & - & - & - & - & - & - & - & & & & & \\
\hline 2008-2009 (0) & - & - & - & - & - & - & - & - & & & & & \\
\hline \multicolumn{14}{|l|}{ Acinetobacter spp. } \\
\hline 2006-2007 (44) & 86.35 & 86.36 & 86.36 & 68.18 & 93.18 & 65.9 & 84.09 & 27.27 & & & & & \\
\hline $2007-2008$ (38) & 94.73 & 97.36 & 89.47 & 81.57 & 76.31 & 68.42 & 84.21 & 47.36 & & & & & \\
\hline 2008-2009 (8I) & 95.06 & 96.29 & 95.06 & 91.35 & 72.83 & 70.37 & 83.95 & 59.25 & & & & & \\
\hline \multicolumn{14}{|l|}{ P. aeruginosa } \\
\hline 2006-2007 (68) & 79.41 & 47.05 & 85.29 & 73.52 & 76.47 & 67.64 & 82.35 & 23.5 & 47.05 & 44.11 & 67.64 & 51.47 & 77.99 \\
\hline $2007-2008$ (57) & 82.45 & 75.43 & 78.94 & 70.17 & 70.17 & 61.40 & 85.96 & 38.59 & 66.66 & 59.64 & 71.92 & 52.63 & 78.94 \\
\hline $2008-2009(8 \mathrm{I})$ & 85.41 & 76.04 & 67.77 & 83.33 & 66.66 & 62.5 & 85.18 & 56.25 & 79.01 & 76.54 & 71.85 & 51.04 & 78.12 \\
\hline
\end{tabular}

$\mathrm{CTX}=$ Ceftriaxone, $\mathrm{CTa}=$ Ceftazidime, $\mathrm{CTi}=$ Ceftizoxime, $\mathrm{AC}=$ Amoxy-Clav, $\mathrm{G}=$ Gentamicin, AK = Amikacin, CF = Ciprofloxacin, Mr = Meropenem, PC = Piperacillin, PT = Piperacillin tazobactom, Az = Aztreonam, NT = Netilmycin, Of = Ofloxacin.; Figures in parenthesis indicate number of isolate. 
of resistance to third-generation cephalosporins and amoxycillin/clavulanic acid during phase II (20062009). During 2006-2007, piperacillin and piperacillin/ tazobactam were found to be relatively effective against $P$. aeruginosa with susceptibility ranging from 52.95 to $55.89 \%$, but susceptibility rapidly decreased to 20.99 and $23.46 \%$, respectively, in 2008-2009. Another important observation of this study was increasing sensitivity trend of aminoglycosides against majority of GNB. During phase I, almost $70-80 \%$ of isolates were resistant to aminoglycosides, whereas during phase II about 50-60\% isolates were resistant to aminoglycosides. Navneeth et $a l^{[4]}$ and Jafari et $a{ }^{[7]}$ also reported increased sensitivity to aminoglycosides against GNBs. Kumari et al reported in their study on analysis of resistance pattern of tracheal and bronchial GNB isolates from the lower respiratory tract specimens of the ICU-admitted patients, the highest mean resistance to cefazolin $(98.8 \%)$ and ampicillin $(97.6 \%)$ while the lowest mean resistance to amikacin (48.5\%). In our study, there was increasing trend of resistance to cephalosporins and amoxycillin/clavulanic acid and declining trend of resistance to aminoglycosides might be due to the selective influence of extensive usage of cephalosporins and amoxycillin/clavulanic acid when compared with aminoglycosides. During the year 2006-2007, meropenem was found to be quite effective against all the GNB with sensitivity ranging from 75 to $90 \%$. Shehabi et al ${ }^{[12]}$ and Lokhart et al ${ }^{[10]}$ also reported carbapenems as the most effective drug for patients in ICUs, but we observed constant decrease in sensitivity during 2007-2009. Carbapenems are frequently used as the last choice in treating serious infections caused by GNB, and there is diminishing number of new antimicrobial drugs in the pharmaceutical pipeline so the observation of this study is of great clinical concern.

We conclude that gram-negative organisms are the predominant pathogens causing LRTI in ICU patients. The increasing trend of resistance in gram-negative organisms is very disturbing. Judicious use of older and newer antimicrobial agents is essential to prevent the emergence of multidrug-resistant bacteria in the ICU. The development of newer therapeutic alternatives to address the problem of MDR GNBs is needed.

\section{References}

1. Vincent JL, Bihari DJ, Suter PM, Bruining HA, White J, NicolasChanoin MH, et al. The prevalence of nosocomial infection in intensive care units in Europe. JAMA 1995;274:639-44.

2. Chastre J, Fagon JY. Ventilator-associated pneumonia. Am J Respir Crit Care Med 2002;165:867-903.

3. Verbist L. Epidemiology and sensitivity of 8625 ICU hematology/ oncology bacterial isolates in Europe. Scand J Infect Dis 1993;91:14-24.

4. Navaneeth BV, Belwadi MR. Antibiotic resistance among gram-negative bacteria of lower respiratory tract secretion in hospitalized patients. Indian J Chest Dis Allied Sci 2002;44:173-6.

5. Gonlugur U, Bakici MZ, Akkurt I, Efeoglu T. Antibiotic susceptibility patterns among respiratory isolates of Gram negative bacilli in Turkish University Hospital. BMC Microbiol 2004;4:32-4.

6. Mukhopadhyay C, Bhargava A, Ayyagari A. Role of mechanical ventilation and development of multidrug resistant organisms in hospital acquired pneumonia. Indian J Med Res 2003;118:229-35.

7. Jafari NJ, Ranjbar R, Haghi-Ashtiani MT, Abedini M, Izadi M. The study of prevalence and antimicrobial susceptibility of tracheal bacterial strains isolated from pediatric patients. Pak J Biol Sci 2009;12:455-8.

8. Forbes BA, Sahm DF, Weissfeld AS, editors. Bacterial identification flow charts and schemes: A Guide to Part III. In: Bailey and Scott's diagnostic microbiology. 12th ed. Missouri: Mosby Elsevier; 2007. p. 251-3.

9. Clinical and Laboratory Standard Institute. Performance standards for antimicrobial susceptibility: Sixteenth informational supplement. Wayne, PA, USA: CLSI: 2006. M100-S16.

10. Lockhart SR, Abramson MA, Beekmann SE, Gallagher G, Riedel S, Diekema DJ, et al. Antimicrobial resistance among gram-negative bacilli causing infections in intensive care unit patients in the United States between 1993 and 2004. J Clin Microbiol 2007;45:3352-9.

11. Kumari HB, Nagarathna S, Chandramuki A. Antimicrobial resistance pattern among aerobic gram negative bacilli of lower respiratory tract specimens of intensive care unit patients in a neurocentre. Indian $\mathrm{J}$ Chest Dis Allied Sci 2007;49:19-22.

12. Shehabi AA, Baadran I. Microbial infection and antibiotic resistance patterns among Jordanian intensive care patients. East Mediterr Health J 1996;2:515-20.

How to cite this article: Gagneja D, Goel N, Aggarwal R, Chaudhary U. Changing trend of antimicrobial resistance among gram-negative bacilli isolated from lower respiratory tract of ICU patients: A 5-year study. Indian J Crit Care Med 2011;15:164-7

Source of Support: Nil, Conflict of Interest: None declared. 